\title{
openheart Nocturnal respiratory rate as a predictor of mortality in patients with acute coronary syndrome
}

\author{
Christian Eick, Patrick Groga-Bada, Kathrin Reinhardt, Martin Duckheim, \\ Lars Mizera, Katharina Böhm, Nina Götz, Meinrad Gawaz, Christine Zürn
}

To cite: Eick C, Groga-Bada P, Reinhardt K, et al. Nocturnal respiratory rate as a predictor of mortality in patients with acute coronary syndrome. Open Heart 2018;5:e000887. doi:10.1136/ openhrt-2018-000887

Received 22 June 2018 Revised 28 August 2018 Accepted 26 September 2018

D) Check for updates

(c) Author(s) (or their employer(s)) 2018. Re-use permitted under CC BY-NC. No commercial re-use. See rights and permissions. Published by BMJ.

Abteilung Innere Medizin III, Department of Cardiology, Eberhard-Karls-Universität Tübingen, Tübingen, Germany

Correspondence to Dr Christian Eick; christian. eick@med.uni-tuebingen.de

\section{ABSTRACT}

Background Patients with acute coronary syndrome (ACS) are at risk especially in the period shortly after the event. Alterations in respiratory control have been associated with adverse prognosis. The aim of our study was to assess if the nocturnal respiratory rate (NRR) is a predictor of mortality in patients with ACS presenting in the emergency department.

Methods Clinically stable consecutive patients with ACS aged $\geq 18$ years were prospectively enrolled. The Global Registry of Acute Coronary Events (GRACE) score and left ventricular ejection fraction (LVEF) were assessed for all patients. The average NRR over a period of 6 hours was determined by the records of the surveillance monitors in the first night after admission. Primary and secondary endpoints were intrahospital and 2 years all-cause mortality, respectively.

Results Of the 860 patients with ACS, 21 (2.4\%) died within the intrahospital phase and 108 patients $(12.6 \%)$ died within the subsequent 2 years. The NRR was a significant predictor of both endpoints and was independent from the GRACE score and LVEF. Implementing the NRR into the GRACE risk model leads to a significant increase of the C-statistics especially for prediction of intrahospital mortality.

Conclusion The NRR is an independent predictor of mortality in patients with ACS.

\section{INTRODUCTION}

Patients with acute coronary syndrome (ACS) are at most risk of mortality during in the first days after the event. All patients with ACS should therefore undergo early risk stratification to identify candidates who are likely to benefit from an intensified treatment and monitoring. Currently, identification of high-risk patients is based on the finding of a reduced left ventricular ejection fraction (LVEF) or by applying score systems such as the GRACE (Global Registry of Acute Coronary Events) risk score. ${ }^{12}$ However, risk stratification of unselected patients with ACS by LVEF $<30 \%$ can only identify a minor part of patients who die after the ACS and the GRACE risk score has its value especially in

\section{Key messages}

What is already know about this subject?

- Previous studies indicate that alterations in respiratory control predict mortality in postinfarction patients.

What does this study add?

- We tested if assessment of the nocturnal respiratory rate (NRR) obtained from routine surveillance monitors immediately after the index event enables a quick and early risk stratification in a large population of all-comers with ACS presenting in the emergency department.

How might this impact on clinical practice?

- The assessment of NRR offers additional prognostic information in patients with ACS for the prediction of mortality.

- This information was obtained with a simple, inexpensive, quick and readily available technology which could be easily implemented in existing monitoring devices.

patients with non-ST-elevation myocardial infarction (NSTEMI). ${ }^{12}$

Experimental and clinical studies indicate that in patients with cardiovascular diseases important prognostic information can be derived from the functional status of the cardiac autonomic nervous system. In the setting of acute myocardial infarction, loss of vagal activity has been linked to increased mortality risk. ${ }^{3}$ Also the respiratory rate is strongly modulated by the autonomic nervous system. Previous studies indicate that alterations in respiratory control predict mortality in postinfarction patients. ${ }^{56}$

In the present study, we tested if assessment of the nocturnal respiratory rate (NRR) obtained from routine surveillance monitors immediately after the index event enables a quick and early risk stratification in a large population of all-comers with ACS presenting in the emergency department. 


\section{METHODS}

\section{Participants}

Consecutive patients $\geq 18$ years were prospectively enrolled between November 2010 and December 2012 having been admitted to the emergency department (which includes the Chest Pain Unit) of the University of Tübingen, Germany, with ACS. ACS was defined as either presence of ST-elevation myocardial infarction (STEMI), NSTEMI or unstable angina pectoris (UAP) according to current guidelines. ${ }^{7-10}$ Clinically unstable patients with cardiogenic shock who required invasive or non-invasive mechanical ventilation were admitted directly to the intensive care unit and were not included in our study. ${ }^{11}$

\section{Assessment of NRR}

Patients were monitored (DASH 4000, General Electrics, Fairfield, Connecticut, USA; sample frequency, $100 \mathrm{~Hz}$ ) for a period of at least 48 hours. Monitoring was performed under routine clinical conditions at the intensive coronary care unit, after performing the acute medical care in the emergency room and catheter lab. The ECG channel of the surveillance monitors was stored on a central server. The respiratory rate was extracted from the ECG recording. Technical details of the methodology of assessment of the respiratory rate from ECG recordings have been described elsewhere. ${ }^{12}$ To calculate the average NRR, the period from 0:00 to 6:00 from the first night after complete admission was used. In addition, we calculated the NRR from shorter recordings, after just 1 hour (0:00 to 01:00) and 2 hours (0.00 to 02.00$)$.

\section{Assessment of LVEF}

The LVEF was determined directly on admission of the patient either by transthoracic echocardiography by the biplane Simpson's method or by left ventriculography in patients who were transferred immediately to the cath lab.

\section{Assessment of the GRACE score}

The GRACE score including age, systolic blood pressure, heart rate, Killip classification, serum creatinine, cardiac arrest at admission, ST-segment deviation and cardiac biomarkers was calculated as previously described. ${ }^{2}$

\section{Study endpoints and follow-up}

The primary study endpoint was the intrahospital all-cause mortality. The secondary endpoint was 2-year all-cause mortality. Intrahospital deaths were assessed via the electronic hospital information system. For assessment of 2-year mortality, patients were contacted by telephone. Two-year follow-up information was available in $95.9 \%$ of the patients. Patients lost to follow-up were censored at the time of last contact.

\section{Statistical analyses}

Continuous variables are presented as median and IQRs and were compared using the Mann-Whitney U test after performing a normality test (Kolmogorov-Smirnov test). Qualitative data are expressed as percentages and were analysed using the $\chi^{2}$ test. Receiver Operating Characteristic (ROC) curves were constructed for risk predictors by plotting 1 - specificity versus sensitivity. ROC curves were quantified by the area under the curve (AUC). To test the difference between ROC curves, bootstrapping was employed based on the creation of pseudo-replicate datasets by random resampling of the dataset $n$ times for error estimation ( $\mathrm{n}=1000$ in this study). The association of risk variables with the endpoints was tested by univariable and multivariable logistic and Cox-regression analyses. Multivariable analyses included NRR as well as the GRACE score and the LVEF. In the analysis for the 2-year mortality, female gender and diabetes were also taken into account. To test the incremental prognostic value of NRR on top of the GRACE risk model, we implemented C-statistic, continuous NRI and the integrated discrimination improvement (IDI) score. Mortality rates were estimated by the Kaplan-Meier method. HRs were presented with $95 \%$ CIs. When used as categorical variable, the NRR was dichotomized at the cut-off value of 16.8 breaths/min. The cut-off value was determined by a method described by Youden et al. ${ }^{13}$ Differences were considered statistically significant when $p$ value was $<0.05$. Statistical analyses were performed using CRAN R V.3.0.1 and SPSS V.21.0.

\section{RESULTS}

During the recruitment period, 860 patients with ACS were prospectively included. Table 1 shows the patients' characteristics. Mean age was 69.7 years, $34.8 \%$ of the patients were female. In 135 patients $(15.7 \%)$ had STEMI, $312(51.2 \%)$ had NSTEMI and $285(33.1 \%)$ suffered from UAP. During NRR recording, $86 \%$ of patients were in sinus rhythm.

Twenty-one patients $(2.4 \%)$ died during the hospital stay. All patients who experienced intrahospital death died due to a cardiovascular reason. In total, 108 patients $(12.6 \%)$ died within 2 years after the index event. The NRR was significantly higher in patients who died during the hospital phase or within the subsequent 2 years than in survivors (18.0 (3.9) breaths/min vs 15.3 (2.4) breaths/min and 16.9 (3.7) breaths/min vs 15.2 (2.2) breaths/min, respectively, $\mathrm{p}<0.001$ for both). Significant differences were also noted for the GRACE score for the primary and secondary endpoint, respectively, and for LVEF (table 2). In addition, patients who died within the hospital stay were significantly older and were more likely to suffer from renal insufficiency. Patients who died within the subsequent 2 years after NRR assessment were of higher age, were more often diabetics, had an impaired renal function and were more often females.

The NRR yielded AUCs of 0.806 (95\% CI 0.725 to $0.887)$ and $0.676(0.616-0.736)$ for the primary and secondary endpoints, respectively $(\mathrm{p}<0.001$ for both, figure 1). Figure 2 shows cumulative mortality rates of patients stratified by NRR. The 206 patients with NRR 
Table 1 Clinical characteristics and outcomes of the study cohort

\begin{tabular}{|lc|}
\hline Variable & $\begin{array}{l}\text { Study population } \\
\text { (n=860) }\end{array}$ \\
\hline Age (years) & $72.0(18.0)$ \\
\hline Female & $299(34.8 \%)$ \\
\hline Left ventricular ejection fraction (\%) & $50.0(20.0)$ \\
\hline Medical history & \\
\hline Myocardial infarction & $270(31.4 \%)$ \\
\hline Congestive heart failure & $216(25.1 \%)$ \\
\hline Atrial fibrillation & $195(22.7 \%)$ \\
\hline Stroke & $87(10.1 \%)$ \\
\hline Peripheral arterial disease & $123(14.3 \%)$ \\
\hline Chronic renal insufficiency & $181(21 \%)$ \\
\hline Percutaneous coronary intervention & $223(25.9 \%)$ \\
\hline Coronary artery bypass graft & $86(10 \%)$ \\
\hline Arterial hypertension & $714(83.0 \%)$ \\
\hline Diabetes mellitus & $270(31.4 \%)$ \\
\hline Hyperlipidaemia & $406(47.2 \%)$ \\
\hline Smoking & $333(38.7 \%)$ \\
\hline Family history of coronary artery disease & $218(25.3 \%)$ \\
\hline STEMl & $135(15.7 \%)$ \\
\hline NSTEMl & $312(51.2 \%)$ \\
\hline UAP & $285(33.1 \%)$ \\
\hline In-hospital coronary angiography & $794(92.3 \%)$ \\
\hline In-hospital percutaneous coronary & $780(90.7 \%)$ \\
\hline intervention & $21(2.4 \%)$ \\
\hline 2-years mortality & $108(12.6 \%)$ \\
\hline
\end{tabular}

Chronic renal insufficiency, GFR $<90 \mathrm{~mL} / \mathrm{min}$; NSTEMI, non-STelevation myocardial infarction; STEMI, ST-elevation myocardial infarction; UAP, unstable angina pectoris.

$\geq 16.8$ breaths $/$ min had an adverse prognosis with a 2-year mortality rate of $27.2 \%$ while the 654 patients with NRR $<16.8$ breaths/min had a significantly better prognosis with a 2-year mortality rate of only $8.0 \%$ ( $<<0.001$ for overall difference).

We also calculated the NRR from shorter recording intervals. For 1 hour the NRR was also significantly higher in patients who died during the hospital phase or within the subsequent 2 years than in survivors (16.7 (2.7) breaths/min vs 14.5 (2.4) breaths/min and 16.0 (3.5) breaths/ $\mathrm{min}$ vs 14.4 (2.3) breaths/min, respectively, $\mathrm{p}<0.001$ for both). The AUC was 0.781 and 0.668 for the primary and secondary endpoints.

For 2 hours the NRR was also significantly higher in patients who died during the hospital phase or within the subsequent 2 years than in survivors (16.5 (3.0) breaths/ min vs 14.5 (2.3) breaths/min and 15.9 (3.5) breaths/ min vs 14.4 (2.2) breaths/min, respectively, $\mathrm{p}<0.001$ for both). The AUC was 0.787 and 0.664 for the primary and secondary endpoints.

In our cohort, 449 of the 860 patients underwent percutaneous coronary intervention (PCI) before NRR assessment, 331 patients underwent $\mathrm{PCI}$ in the period after the NRR assessment and 80 patients did not undergo PCI. Significantly higher NRR values can be seen in non-survivors of all subgroups except for the patients without PCI with regard to 2-year mortality. Especially in patients treated by PCI after NRR assessment (331 patients, two intrahospital deaths), the NRR showed a very good C-statistic with an AUC of 0.977 regarding the intrahospital mortality (table 3 ).

In a further subgroup analysis, we investigated the NRR in patients with STEMI, NSTEMI and UAP. In all subgroups, except for the 2-year mortality in patients with UAP, non-survivors show significantly higher NRR values compared with survivors (table 4).

Table 5shows univariable and multivariable analyses of NRR, the GRACE score and LVEF for prediction of both endpoints. NRR proved to be an independent risk factor yielding a HR of 1.35 (95\% CI 1.09 to $1.68, \mathrm{p}=0.006$ ) for intrahospital mortality and 1.15 (95\% CI 1.05 to 1.26 , $\mathrm{p}=0.002$ ) for 2-year mortality, respectively. Implementing NRR into the GRACE risk model led to a significant increase of the C-statistics for prediction of intrahospital mortality from 0.842 (95\% CI 0.740 to 0.944$)$ to 0.876 ( $95 \%$ CI 0.790 to $0.961 ; \mathrm{p}=0.02$ for difference, continuous NRI 0.556, $\mathrm{p}=0.01$, IDI 0.022: $\mathrm{p}<0.01$, figure $3 \mathrm{~A}$ ). For prediction of 2-year mortality only the continuous NRI showed significant improvement from 0.776 (95\% CI 0.731 to 0.822 ) to 0.784 (95\% CI 0.737 to $0.830 ; \mathrm{p}=0.95$ for difference, continuous NRI $0.313, \mathrm{p}<0.01$, IDI 0.0175 ; $\mathrm{p}=0.286$, figure $3 \mathrm{~B}$ ).

\section{DISCUSSION}

The present study shows that the average NRR during the first night following hospital admission provides powerful prognostic information regarding intrahospital mortality in all-comers with ACS presenting in the emergency department. The prognostic value is independent from established risk predictors such as the GRACE score and LVEF. In addition, the NRR significantly improved the GRACE risk model. Regarding 2-year mortality, NRR also proved to be an independent risk factor but only improved the GRACE risk model to a weaker extent.

Current guidelines recommend monitoring of patients with ACS as these patients are at substantial risk of death due to malignant arrhythmias, reinfarction or progressive heart failure. ${ }^{14}$ Some patients, however, are likely to benefit from an intensified treatment and monitoring. The identification of high-risk patients at an early stage after admission would be of great clinical interest. Established methods for risk assessment in patients with ACS are clinical risk scores such as the GRACE score or LVEF. ${ }^{115}$ However, prognostic evaluation using these approaches has limitations and risk markers complementary to the 
Table 2 Characteristics of survivors and non-survivors of the intrahospital phase

\begin{tabular}{|c|c|c|c|c|c|c|}
\hline & \multicolumn{3}{|l|}{ IHM } & \multicolumn{3}{|l|}{ 2YM } \\
\hline & $\begin{array}{l}\text { Survivors } \\
(n=839)\end{array}$ & $\begin{array}{l}\text { Non-survivors } \\
(n=21)\end{array}$ & P values & $\begin{array}{l}\text { Survivors } \\
(n=752)\end{array}$ & $\begin{array}{l}\text { Non-survivors } \\
(n=108)\end{array}$ & $P$ values \\
\hline Age (years) & $72.0(18.0)$ & $81.0(12.0)$ & 0.002 & $71.0(18.0)$ & $80.0(11.0)$ & $<0.001$ \\
\hline Female & $290(34.6 \%)$ & $9(42.9 \%)$ & 0.488 & $247(32.8 \%)$ & $52(48.1 \%)$ & 0.002 \\
\hline GRACE score (points) & $122(44)$ & $182(40)$ & $<0.001$ & $119(43)$ & $157(50)$ & $<0.001$ \\
\hline LVEF (\%) & $50(20)$ & $30(25)$ & 0.005 & $50(20)$ & $40(25)$ & $<0.001$ \\
\hline Diabetes mellitus & $261(31.1 \%)$ & $9(42.9 \%)$ & 0.245 & $218(29.0 \%)$ & $52(48.1 \%)$ & $<0.001$ \\
\hline Renal insufficiency & $170(20.3 \%)$ & $11(52.4 \%)$ & $<0.001$ & $135(18.0 \%)$ & $46(42.6 \%)$ & $<0.001$ \\
\hline NRR (breaths/min) & $15.3(2.4)$ & $18.0(3.9)$ & $<0.001$ & $15.2(2.2)$ & $16.9(3.7)$ & $<0.001$ \\
\hline
\end{tabular}

GRACE, Global Registry of Acute Coronary Events; IHM, intrahospital mortality; LVEF, left ventricular ejection fraction; NNR, nocturnal respiration rate; $2 \mathrm{YM}, 2$-year mortality.

established methods are warranted. The optimal risk marker would be easily and quickly determinable, inexpensive, without demands on technical equipment or on patient cooperation. ${ }^{16}$ Most of these requirements apply to the NRR: the determination of NRR can be carried out using most surveillance monitors and without additional expense. The nocturnal determination off NRR allows standardised test conditions, also without any extra effort and without specific patient cooperation.

The NRR can be assessed by different methods through routine surveillance monitors. In this study, the respiratory rate was calculated from the ECG channel because not all surveillance monitors support other methods of respiration rate assessment. ${ }^{12}$ This method is therefore applicable to monitoring stations with basic technical equipment. Since all patients with ACS require monitoring especially in the first $24-48$ hours, ${ }^{14}$ the determination of NRR could be integrated into routine clinical practice without significant additional expense.

We were able to show that the average NRR during the first night after admission provides additional prognostic information, especially with respect to the intrahospital mortality. On the long term, the NRR might therefore be helpful for clinical decisions such as prolonged monitoring and intensified medical, interventional and devicebased treatment of high-risk patients with ACS.

Since the time of revascularisation can have a considerable effect on NRR and on outcome, we have carried out subgroup analyses of patients who underwent PCI before NRR assessment, patients who underwent PCI after NRR assessment and patients who did not undergo PCI. A significantly higher NRR can be seen in the non-survivors of all subgroups except for patients without PCI with regard to 2-year mortality. A very good C-statistic was found for patients in whom the NRR was assessed prior to revascularisation with regard to intrahospital mortality. These patients might have benefited from an earlier invasive diagnosis and therapy. However, the low event rate in this subgroup only allows to draw this conclusion to a limited extent.

The fact that the respiratory rate provides prognostic information in postinfarction patients has already been shown in other studies. ${ }^{51718}$ The NRR seems to be particularly suitable because of its assessment under standardised conditions in the night compared with daytime measurements. ${ }^{6}$ Other studies used standard 24-hour
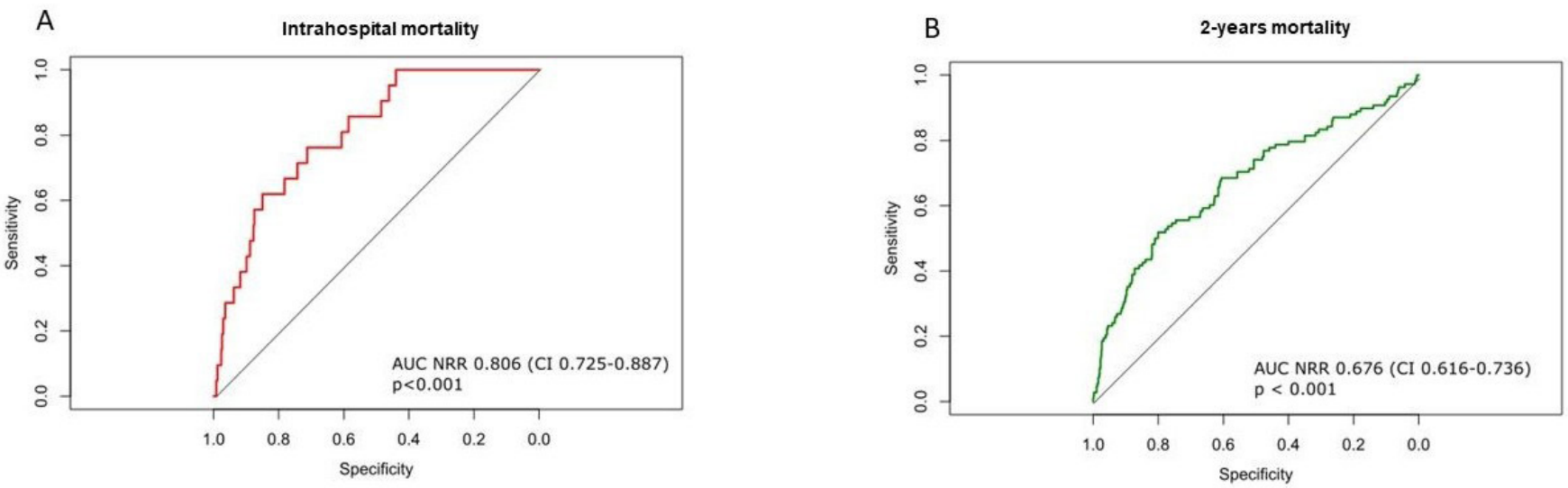

Figure 1 Receiver-operator characteristic curves for prediction of the primary and secondary endpoint by the nocturnal respiratory rate (NNR) in patients with acute coronary syndrome. AUC, area under the curve. 


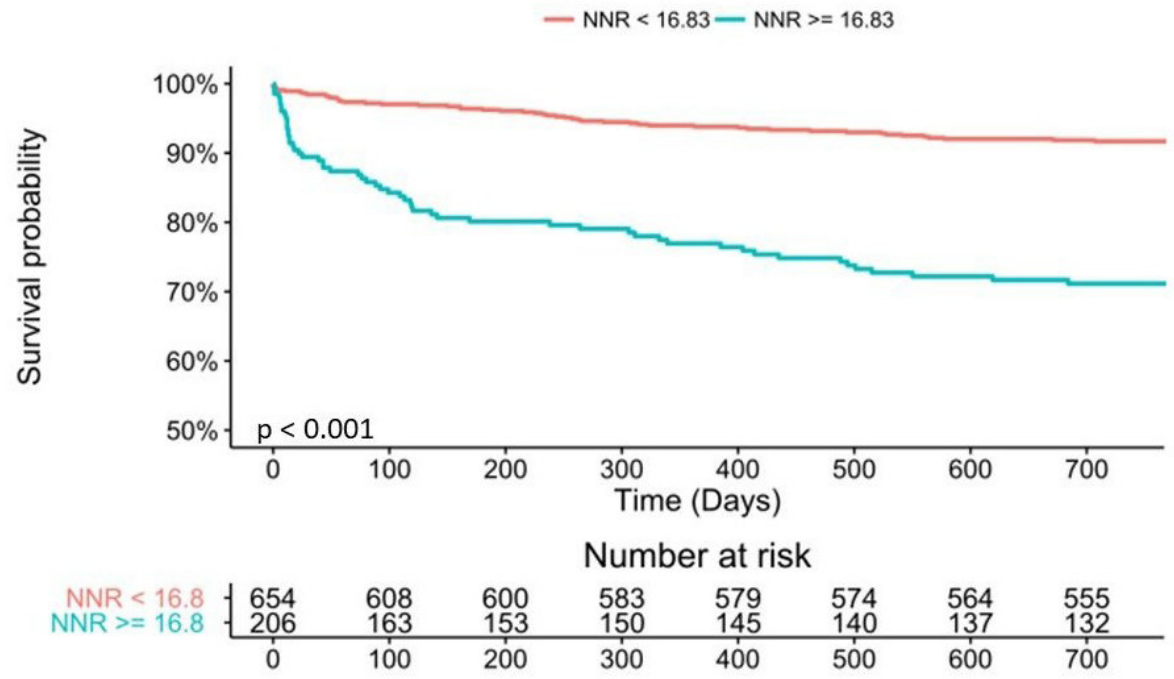

Figure 2 Cumulative mortality rate of patients stratified by the nocturnal respiratory rate (NNR) $<16.8$ breaths/min and $\geq 16.8$ breaths/min, respectively.

Holter recordings or short records of 10 min respiratory rate at rest within an extended period for prediction of mortality after myocardial infarction. ${ }^{5617}$ The difference of our study is first that we have determined the NRR in the routine clinical setting of the emergency department via standard patient monitors without any additional diagnostic tools. Second, the time of NRR assessment is clearly defined in our study, that is, the first night after hospital admission. Thus, by the presented setting objective prognostic information can be obtained shortly after the clinical event, which can be useful in addition to other risk factors for immediate planning of further treatment of the individual patient. Third, other studies have assessed an association between respiratory rate and post-MI prognosis. We have focused on a broader spectrum of all-comers with ACS including patients with UAP.

The respiratory rate is strongly influenced by the balance of the autonomic nervous system. Increased respiratory rate at admission may therefore by a sign of altered autonomic control of respiratory activity due

\begin{tabular}{llrllllllll}
\hline \multicolumn{1}{l}{ Table 3} & \multicolumn{1}{l}{ Subgroup analysis depending on the time of therapy and the modality of therapy } \\
\hline Treatment & IHM & \multicolumn{1}{c}{ N } & NRR & P values & AUC & 2YM & N & NRR & P values & AUC \\
\hline No PCl & No & 71 & $15.8 \pm 1.7$ & $<0.001$ & 0.837 & No & 58 & $15.9 \pm 1.7$ & 0.228 & 0.588 \\
& Yes & 9 & $18.3 \pm 1.9$ & & & Yes & 22 & $16.6 \pm 2.2$ & & \\
PCl before NRR & No & 439 & $15.5 \pm 1.9$ & 0.007 & 0.748 & No & 401 & $15.4 \pm 1.8$ & $<0.001$ & 0.704 \\
assessment & Yes & 10 & $17.1 \pm 1.8$ & & & Yes & 48 & $17.1 \pm 2.5$ & & \\
PCl after NRR & No & 329 & $15.5 \pm 2.1$ & 0.02 & 0.977 & No & 293 & $15.4 \pm 2.1$ & $<0.001$ & 0.665 \\
assessment & Yes & 2 & $20.4 \pm 0.4$ & & & Yes & 38 & $16.8 \pm 2.6$ & &
\end{tabular}

AUC, IHM, intrahospital mortality; NRR, nocturnal respiration rate; NSTEMI, non-ST-elevation myocardial infarction; PCI, percutaneous coronary intervention; STEMI, ST-elevation myocardial infarction; UAP, unstable angina pectoris; 2YM, 2-year mortality.

\begin{tabular}{lllllllllll}
\multicolumn{2}{l}{ Table 4} & \multicolumn{1}{l}{ Subgroup analysis depending on the type of ACS } \\
\hline ACS group & IHM & N & NRR & P values & AUC & 2YM & N & NRR & P values & AUC \\
\hline STEMI & No & 131 & $15.5 \pm 1.8$ & 0.018 & 0.847 & No & 120 & $15.4 \pm 1.6$ & 0.006 & 0.717 \\
& Yes & $4(3.0 \%)$ & $17.6 \pm 1.3$ & & & Yes & $15(12.5 \%)$ & $16.9 \pm 12.5$ & & \\
\multirow{2}{*}{ NSTEMI } & No & 424 & $15.9 \pm 2.1$ & $<0.001$ & 0.747 & No & 367 & $15.8 \pm 2.0$ & $<0.001$ & 0.675 \\
& Yes & $16(3.7 \%)$ & $17.9 \pm 2.1$ & & & Yes & $73(19.9 \%)$ & $17.2 \pm 2.4$ & & \\
UAP & No & 284 & $15.0 \pm 1.8$ & 0.007 & 1.000 & No & 265 & $15.0 \pm 1.8$ & 0.266 & 0.575 \\
& Yes & $1(0.3 \%)$ & 20.6 & & & Yes & $20(7.5 \%)$ & $15.6 \pm 2.4$ & &
\end{tabular}

AUC, area IHM, intrahospital mortality; NRR, nocturnal respiration rate; NSTEMI, non-ST-elevation myocardial infarction; STEMI, ST-elevation myocardial infarction; UAP, unstable angina pectoris; 2YM, 2-year mortality. 
Table 5 Univariable and multivariable binary logistic regression and Cox regression analysis for prediction of intrahospital mortality and 2-year mortality

\begin{tabular}{|c|c|c|c|c|c|c|}
\hline \multicolumn{7}{|l|}{ IHM } \\
\hline \multirow[b]{2}{*}{ Variable } & \multicolumn{3}{|c|}{ Univariable binary logistic regression } & \multicolumn{3}{|c|}{ Multivariable binary logistic regression } \\
\hline & HR (95\% Cl) & Wald & $P$ values & HR (95\% Cl) & Wald & $P$ values \\
\hline GRACE score & 1.04 (1.03 to 1.06$)$ & 34.7 & $<0.001$ & 1.04 (1.02 to 1.68$)$ & 20.0 & $<0.001$ \\
\hline NRR (breaths/min) & 1.63 (1.34 to 1.98$)$ & 24.1 & $<0.001$ & 1.35 (1.09 to 1.68$)$ & 7.5 & 0.006 \\
\hline LVEF (\%) & 0.94 (0.90 to 0.98 ) & 10.1 & 0.002 & 0.98 (0.94 to 1.02$)$ & 1.1 & 0.298 \\
\hline \multicolumn{7}{|l|}{ 2YM } \\
\hline \multirow[b]{2}{*}{ Variable } & \multicolumn{3}{|c|}{ Univariable Cox regression } & \multicolumn{3}{|c|}{ Multivariable Cox regression } \\
\hline & HR (95\% Cl) & Wald & $P$ values & HR (95\% Cl) & Wald & $P$ values \\
\hline GRACE score & $1.03(1.02$ to 1.03$)$ & 113.9 & $<0.001$ & $1.02(1.02$ to 1.03$)$ & 53.8 & $<0.001$ \\
\hline Female & $1.80(1.23$ to 2.6$)$ & 9.1 & 0.02 & 0.86 (0.70 to 1.04$)$ & 2.4 & 0.123 \\
\hline NRR (breaths/min) & 1.35 (1.24 to 1.47$)$ & 50.0 & $<0.001$ & 1.15 (1.05 to 1.26$)$ & 9.9 & 0.002 \\
\hline LVEF (\%) & 0.94 (0.93 to 0.96 ) & 50.7 & $<0.001$ & 0.97 (0.95 to 0.98$)$ & 13.6 & $<0.001$ \\
\hline Diabetes mellitus & 0.48 (0.33 to 0.71$)$ & 14.21 & $<0.001$ & 0.62 (0.43 to 0.91$)$ & 6.0 & 0.014 \\
\hline
\end{tabular}

GRACE, Global Registry of Acute Coronary Events; IHM, intrahospital mortality; LVEF, left ventricular ejection fraction; NNR, nocturnal respiration rate; 2 YM, 2-year mortality.

to enhanced sympathetic activity and vagal withdrawal in ACS and also maybe due to progressive pulmonary congestion. ${ }^{4}$ Other parameters evaluating the function of the autonomic nervous system, such as various parameters of heart rate variability, are also strong risk predictors in patients with ACS. ${ }^{49-22}$ For assessment of these parameters, however, the presence of sinus rhythm is mandatory which is not needed for determination of NRR.

The limitations of our study need to be recognised. First, we cannot compare the prognostic information of NRR with parameters of heart rate variability since the study cohort includes patients with atrial fibrillation. However, as atrial fibrillation in patients with ACS is often prevalent, risk stratification by NRR which can be performed irrespective of the patient's heart rhythm is favourable. Second, the presence of motion artefacts and other technical failures could affect the determination of the respiratory rate from the ECG channel. Further underlying respiratory pattern could affect the NRR. The calculation of the average respiratory rate over a period of 6 hours, however, should reduce the influence of corresponding disturbances. The choice of the period from 0:00 to 6:00 was due to the consideration that during this time the patient is most likely asleep; however, there was no monitoring of the sleep state. However, we consider that the chosen nightly episode is the optimal time for the standardised determination of the respiratory rate since the external influences are the lowest at this time. We also calculated the NRR from shorter recording intervals (1 and 2 hours). Again, there were highly significant differences with respect to both endpoints. However, the respective $\mathrm{C}$-statistic was better with a longer recording time (6 hours). We interpret this by the fact that disturbances have less effects with longer recording time. In
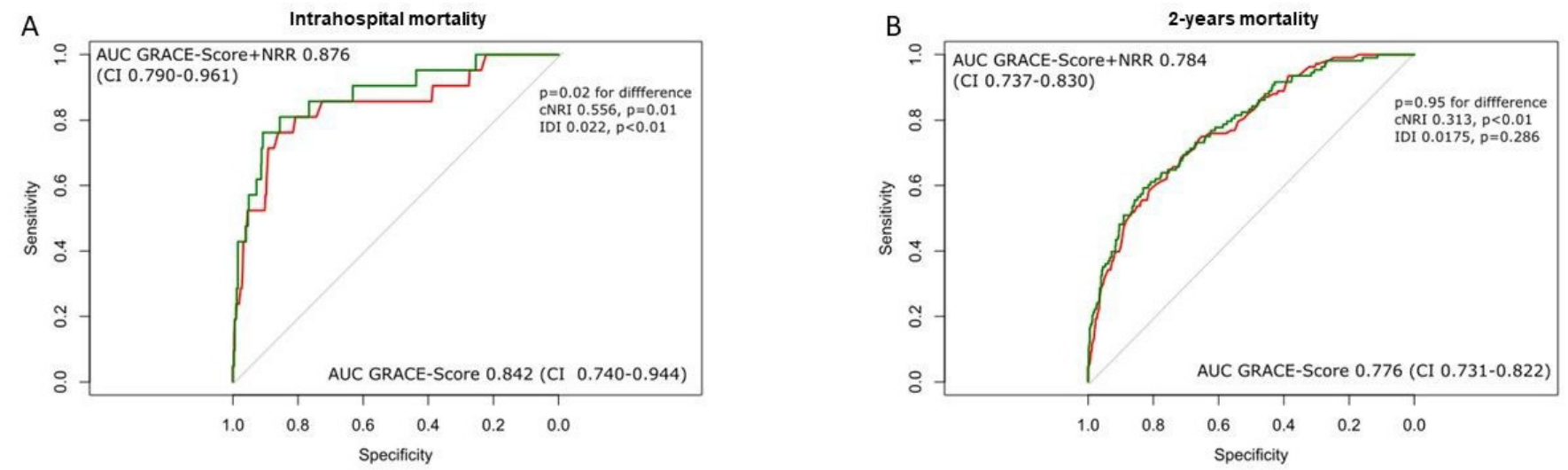

Figure 3 Receiver-operator characteristic curves for prediction of the primary and secondary endpoint in patients with acute coronary syndrome. (A, B)The GRACE score as well as the combination of the GRACE score and the nocturnal respiratory rate (NRR). AUC, area under the curve. 
addition, specific respiratory disorders that may affect the results are also better detected with longer recording time.

Third, the selected cut-off for NRR of $16.8 / \mathrm{min}$ was determined retrospectively and was not derived from pathophysiological reasoning or previous literature data. Fourth, ACS is composed of multiple types of infarction and ischaemia which have variable effects on heart and vascular baroceptors and chemoceptors and finally also on the NRR. However, we performed subgroup analyses on patients with STEMI, NSTEMI and UAP and non-survivors of all ACS subsets presented with significantly higher NRR values except patients with UAP who died within 2 years. Fifth, we cannot provide systematic information about the exact time of the onset of ACS and with regard to drugs given in the first hours of ACS which both may have an effect on NRR. Sixth, LVEF was assessed by echocardiography or by left ventriculography. However, studies report a sufficient correlation of both modalities to assess LVEF. ${ }^{23}$

Finally, further studies are needed to show that incorporation of the NRR into the risk stratification of patients with ACS and clinical decision-making contributes to an improved prognosis.

\section{CONCLUSIONS}

The NRR is an independent predictor of intrahospital mortality in patients with ACS. The assessment of NRR offers additional prognostic value to GRACE score and LVEF in patients with ACS for the prediction of in-hospital mortality. This information was obtained with a simple, inexpensive, quick and readily available technology which could be easily implemented in existing monitoring devices.

Contributors CE developed the theoretical formalism, performed the analytic calculations and performed the numerical simulations. CE, PG-B, KR, MD, LM, KB and NG have performed the collection of data MG and CSZ verified the analytical methods and supervised the findings of this work. All authors discussed the results and contributed to the final manuscript.

Funding This study was kindly supported by the Geschwister-Kessel-Stiftung, Langenenslingen, Germany.

Competing interests None declared.

Patient consent Not required.

Ethics approval University of Tuebingen.

Provenance and peer review Not commissioned; externally peer reviewed.

Data sharing statement No additional data are available.

Open access This is an open access article distributed in accordance with the Creative Commons Attribution Non Commercial (CC BY-NC 4.0) license, which permits others to distribute, remix, adapt, build upon this work non-commercially, and license their derivative works on different terms, provided the original work is properly cited, appropriate credit is given, any changes made indicated, and the use is non-commercial. See: http://creativecommons.org/licenses/by-nc/4.0/

\section{REFERENCES}

1. Goldberg RJ, Currie K, White K, et al. Six-month outcomes in a multinational registry of patients hospitalized with an acute coronary syndrome (the Global Registry of Acute Coronary Events [GRACE]). Am J Cardiol 2004;93:288-93.

2. Granger CB, Goldberg RJ, Dabbous O, et al. Predictors of hospital mortality in the global registry of acute coronary events. Arch Intern Med 2003;163:2345-53.

3. Schwartz PJ, La Rovere MT, Vanoli E. Autonomic nervous system and sudden cardiac death. Experimental basis and clinical observations for post-myocardial infarction risk stratification. Circulation 1992;85(1 Suppl):I77-91.

4. Kleiger RE, Miller JP, Bigger JT, et al. Decreased heart rate variability and its association with increased mortality after acute myocardial infarction. Am J Cardiol 1987;59:256-62.

5. Barthel $P$, Wensel $R$, Bauer A, et al. Respiratory rate predicts outcome after acute myocardial infarction: a prospective cohort study. Eur Heart J 2013;34:1644-50.

6. Dommasch M, Sinnecker D, Barthel P, et al. Nocturnal respiratory rate predicts non-sudden cardiac death in survivors of acute myocardial infarction. J Am Coll Cardiol 2014;63:2432-3.

7. Thygesen K, Alpert JS, Jaffe AS, et al. Third universal definition of myocardial infarction. J Am Coll Cardiol 2012;60:1581-98.

8. Task Force on the management of ST-segment elevation acute myocardial infarction of the European Society of Cardiology (ESC), Steg PG, James SK, et al. ESC Guidelines for the management of acute myocardial infarction in patients presenting with ST-segment elevation. Eur Heart J 2012;33:2569-619.

9. Hamm CW, Bassand JP, Agewall S, et al. ESC Guidelines for the management of acute coronary syndromes in patients presenting without persistent ST-segment elevation: The Task Force for the management of acute coronary syndromes (ACS) in patients presenting without persistent ST-segment elevation of the European Society of Cardiology (ESC). Eur Heart J 2011;32:2999-3054.

10. Thygesen K, Alpert JS, White HD, et al. Universal definition of myocardial infarction. Eur Heart $J$ 2007;28:2525-38.

11. Eick C, Rizas KD, Meyer-Zürn CS, et al. Autonomic nervous system activity as risk predictor in the medical emergency department: a prospective cohort study. Crit Care Med 2015;43:1079-86.

12. Moody GB, Mark RG, Zoccola A. Derivation of respiratory signals from multi-lead ECGs. Comput Cardiol 1985;12:113-6.

13. Youden WJ. Index for rating diagnostic tests. Cancer 1950;3:32-5.

14. Roffi M, Patrono C, Collet JP, et al. 2015 ESC Guidelines for the management of acute coronary syndromes in patients presenting without persistent ST-segment elevation: Task Force for the Management of Acute Coronary Syndromes in Patients Presenting without Persistent ST-Segment Elevation of the European Society of Cardiology (ESC). Eur Heart J 2016;37:267-315.

15. Rahimi K, Watzlawek S, Thiele H, et al. Incidence, time course, and predictors of early malignant ventricular arrhythmias after nonST-segment elevation myocardial infarction in patients with early invasive treatment. Eur Heart J 2006;27:1706-11.

16. Malik M. Autonomic tests to detect cardiac risk and their clinical practicality. J Cardiovasc Electrophysiol 2011;22:no-30.

17. Sinnecker D, Dommasch M, Barthel P, et al. Assessment of mean respiratory rate from ECG recordings for risk stratification after myocardial infarction. J Electrocardiol 2014;47:700-4.

18. Helmers C. Assessment of 3-year prognosis in survivors of acute myocardial infarction. Br Heart $J$ 1975;37:593-7.

19. Bauer A, Barthel P, Schneider R, et al. Improved Stratification of Autonomic Regulation for risk prediction in post-infarction patients with preserved left ventricular function (ISAR-Risk). Eur Heart $J$ 2009;30:576-83.

20. Bauer A, Kantelhardt JW, Barthel P, et al. Deceleration capacity of heart rate as a predictor of mortality after myocardial infarction: cohort study. Lancet 2006;367:1674-81.

21. Bonnemeier H, Hartmann F, Wiegand UK, et al. Heart rate variability in patients with acute myocardial infarction undergoing primary coronary angioplasty. Am J Cardiol 2000;85:815-20.

22. Rovere MTL, Bigger JT, Marcus FI, et al. Baroreflex sensitivity and heart-rate variability in prediction of total cardiac mortality after myocardial infarction. The Lancet 1998;351:478-84.

23. Gholamrezanezhad A, Mirpour S, Esfehani AF, et al. A correlative study comparing current different methods of calculating left ventricular ejection fraction. Nucl Med Commun 2007;28:41-8. 